\title{
Programming of the lung by early-life infection
}

\author{
P. M. Hansbro ${ }^{1 *}$, M. R. Starkey ${ }^{1}$, R. Y. Kim ${ }^{1}$, R. L. Stevens ${ }^{2}$, P. S. Foster ${ }^{1}$ and J. C. Horvat ${ }^{1}$ \\ ${ }^{1}$ Centre for Asthma and Respiratory Disease and Hunter Medical Research Institute, The University of Newcastle, Newcastle, Australia \\ ${ }^{2}$ Department of Medicine, Harvard Medical School and Brigham and Women's Hospital, Boston, Massachusetts, USA
}

\begin{abstract}
Many important human diseases, such as asthma, have their developmental origins in early life. Respiratory infections in particular may alter the course of asthma and may either protect against or promote the development of this disease. It is likely that the nature of the effects depends on the type and age of infection and is determined by the impact of infection on the immune and respiratory systems. Immunity in early life is plastic and can be moulded by antigen encounter, which may enhance or reinforce the asthmatic phenotype of early life, or induce protective responses. Chlamydial respiratory infections have specific effects and may increase asthma severity in early life by promoting systemic interleukin 13 responses and causing permanent changes in lung structure. Respiratory viral infections, such as those of respiratory syncytial virus and rhinovirus, promote pro-asthmatic responses in early life that contribute to the induction of asthma. By contrast, probiotics or infection or exposure to certain bacteria, such as Streptococcus pneumoniae, may have protective effects in asthma by increasing the numbers and activity of regulatory $\mathrm{T}$ cells. Here, we review the impact of infections on the developmental origins of asthma. Understanding these effects may lead to new therapeutic approaches for asthma that either target deleterious infections or utilize beneficial ones.
\end{abstract}

Received 4 November 2011; Revised 29 December 2011; Accepted 18 January 2012; First published online 14 February 2012

Key words: developmental origins of disease, immunity, infection, lung structure, respiratory

\section{Role of infections in the developmental origins of asthma and other diseases}

Infections in early life have long-term consequences on respiratory and general health. This is because immunity in early life is plastic and can be altered and moulded by factors and events such as exposure to infections and allergens, as well as different diets and obesity. ${ }^{1-8}$ Infections in early life alter immunity by affecting the phenotype of immune responses, especially of $\mathrm{T}$ helper cells, macrophages and other immune and structural cells. ${ }^{9-11}$ Infections may also reduce lung function, which becomes irreversible as the lungs mature during the first 2 years of life. Thus, infections may play a major role in the developmental origins of health and disease. They may either protect against or predispose to the development of many other conditions, for example, asthma. $3,4,10-17$

The nature of immune responses to infectious agents and allergens is the result of coordinated responses of immune cells that regulate each other through complex signalling networks. Innate and adaptive immune cell activity and cytokine release may be critically important in driving pathogenic responses to infections and to allergens. In asthma, T helper type 2 (Th2) cells and the cytokines they release, interleukin (IL)-4, IL-5 and IL-13, mediate pathogenesis (reviewed in $^{18}$ ). IL-4 is necessary for Th2 cell differentiation, promotes immunoglobulin $\mathrm{E}$ production, allergic inflammation and the development of

*Address for correspondence: Professor P. M. Hansbro, Infection and Immunity, The David Maddison Clinical Sciences Building, The University of Newcastle, Cnr King and Watt Sts, Newcastle, NSW 2300, Australia. (Email Philip.Hansbro@newcastle.edu.au) mucus hypersecretion and airway hyper-responsiveness (AHR). IL-5 is required for the development and influx of eosinophils and may contribute to AHR. IL-13 is a critical mediator of Th2 responses and hallmark features of allergic airway disease (e.g. inflammation, mucus hypersecretion and AHR). Antigenpresenting cells (APCs), plasmacytoid and myeloid dendritic cells (DCs) and alveolar macrophages, are innate immune cells that are critical mediators of adaptive responses to infection and are important in the mechanisms that lead to alterations in T-cell programming. APCs recognize pathogens and present antigen as part of the major histocompatibility complexes to $\mathrm{T}$ cells, which drives the development of effector $\mathrm{T}$ cells with particular functions (e.g. protective or destructive). ${ }^{19-21}$ Infections may alter the phenotype of APCs by altering surface molecule expression, inducing $\mathrm{Th} 2$ and inflammatory responses that promote allergic airway diseases, such as asthma.

Numerous pathogens that infect the lung are associated with the development of asthma. Early-life infections with viruses (respiratory syncytial virus, rhinovirus, influenza) and bacteria (Chlamydia and Mycoplasma) have deleterious effects and may drive the development of wheezing, asthma and stress and potentially have systemic effects. ${ }^{3,4,22-24}$ Others, such as probiotics, and as we have shown Streptococcus pneumoniae, may be beneficial and suppress pro-asthmatic responses. $^{12,25-29}$ There has also been substantial recent interest in the role of the entire lung microbiome in the development of asthma. ${ }^{30-32}$ There is an increase in the numbers and species (i.e. the bioburden) of the lung microbiome in asthma, and reduced lung function is related to bioburden and diversity. Nevertheless, the mechanisms that underpin the effects of 
infections remain poorly understood. If we can elucidate the mechanisms, we may be able to develop new treatments for asthma that either target deleterious pathogens or utilize beneficial ones.

\section{The association of chlamydial respiratory tract infections in early life and asthma}

Chlamydial respiratory tract infections (RTIs) are an example of how infections in early life may promote the developmental origins of disease and asthma. RTIs with Chlamydia pneumoniae are very common and frequently cause asymptomatic lung infection and acute respiratory disease in children and adults. At any particular time, 5\% of children and adults have asymptomatic chlamydial respiratory infection. ${ }^{33,34}$ Importantly, $80 \%$ of adults have anti-chlamydial antibodies, indicating that most individuals have been infected at some stage of their lives. ${ }^{35}$ Substantial epidemiological, clinical and experimental evidence associates infection with $C$. pneumoniae with the development and exacerbation of asthma, particularly severe asthma in children and adults. ${ }^{3,13,36-40}$ Recent studies specifically implicate early-life chlamydial infection in the development of reduced lung function and asthma later in life. ${ }^{10,11,13,37-40}$ Early C. pneumoniae infection in children is strongly associated with acute wheezing, disease progression and asthma. ${ }^{13,38,39,41-45}$ Indeed, $50 \%$ and $70 \%$ of children with severe asthma have culturable and detectable C. pneumoniae and/or Chlamydia trachomatis in their lungs, respectively. The role of Chlamydia in asthma is almost certainly underappreciated because of the following: the need to assess lower respiratory tract tissues, difficulties in detection, lack of standardized methods, assessment not being routine in clinical laboratories, infections often being asymptomatic and the effects of infection persisting after the clearance of the bacteria. ${ }^{3,46,47}$ Given that many individuals are infected in the respiratory tract with Chlamydiae, it is likely that 'two hits' are required for asthma development and both environmental and genetic factors are likely to contribute. ${ }^{48}$ Such factors include the status of maturation of the immune system and allergic sensitization. Indeed, some individuals may be genetically predisposed to aberrant immune responses (i.e. Th2 responses) to environmental factors (e.g. chlamydial RTIs) that may inhibit the clearance of infectious agents and promote the development of allergy and asthma. $^{3}$

The mechanisms of the association between early-life chlamydial RTI, reduced lung function and enhanced asthma in later life remain little known and there are currently no effective treatments for infection-associated asthma. It is likely that infection induces permanent immune and structural alterations that play crucial pathogenic roles, ${ }^{49}$ and we have investigated these possibilities (see subsequent sections). In addition, we, and others, have also shown that Chlamydia can infect and activate airway epithelial cells and induce the release of IL-8, IL-13 and granulocyte macrophage-colony-stimulating factor that could enhance airway inflammation and asthma severity. ${ }^{18,50-52}$ Chlamydiae can also infect endothelial cells and induce the release of profibrotic factors such as basic fibroblast growth factor and cytokines such as IL-8 and monocyte chemotactic protein-1, as well as soluble factors that may contribute to inflammation and remodelling in asthma. ${ }^{53-55}$ These bacteria also have the capacity to infect airway smooth muscle cells, ${ }^{56}$ which may alter muscle tone and induce AHR. Chlamydiae may also affect the innervation of the airways and can infect cells of the nervous system such as microglia and astrocytes and promote the release of pro-inflammatory cytokines such as IL-6, which may induce Th2 cell development and expansion, as well as AHR. ${ }^{57}$ Detailed studies that determine how infections promote the development and exacerbation of asthma are required to facilitate the design of effective interventions. ${ }^{58}$

\section{Chlamydial RTI in early life causes permanent alterations in immunity and lung structure and function, and increases the severity of asthma}

Our group, and others, have investigated the mechanisms of how chlamydial RTIs are associated with the induction and increased severity of allergic airway disease in later life using mouse models. ${ }^{10,11,59}$ We developed a model of neonatal chlamydial RTI and combined it with the classic model of ovalbumin-induced allergic airway disease. We used Chlamydia muridarum, which is a natural mouse respiratory pathogen and is the most appropriate chlamydial species to investigate hostpathogen relationships and the impact of infection on allergic airway disease in mice. ${ }^{10,11}$ We infected neonatal mice on the 1 st day of life with 400 inclusion-forming units of $C$. muridarum. The bacteria replicated in the lungs and infection peaked after 10 days, declined after 15 days and was cleared by 21 days. ${ }^{10}$ Infection was mirrored by histopathological evidence of inflammation in the lung. Infection was allowed to resolve and allergic airway disease was induced on day 45 . We then assessed the impact of infection on allergic airway disease. ${ }^{10,11}$ Controls were: (1) infection without allergic airway disease, (2) allergic airway disease without infection and (3) no infection or allergic airway disease.

The induction of allergic airway disease resulted in the development of hallmark features of asthma with increased numbers of mucus-secreting cells around the airways, IL-13 expression in the lungs and increased AHR. Neonatal chlamydial RTI increased the severity of allergic airway disease in later life by further increasing mucus-secreting cell numbers, IL-13 expression and AHR. The mechanisms involved were then investigated. The development of allergic airway disease was also characterized by eosinophilic inflammation of airway tissue, increased Th2 cytokine release (IL-5 and IL-13) from ovalbumin-restimulated $\mathrm{T}$ cells in the lung-draining mediastinal lymph nodes (MLN) and the influx of activated mDCs into the lungs. Surprisingly, all of these pro-inflammatory responses that are normally associated with enhancing allergic airway disease were suppressed by the prior neonatal chlamydial RTI, with 
infection instead inducing a mixed Th2/Th1 response. The effects of infection on systemic DC-induced T-cell responses were then assessed. DCs were isolated from spleens of adult (9-week-old) mice that were infected or sham-exposed as neonates. DCs were pulsed with ovalbumin and cultured with ovalbumin-peptide T-cell receptor-specific transgenic (ovalbumin TCR Tg) T cells. Neonatal infection resulted in the increased release of IL-13 in DC and T-cell co-cultures. IL-13 can induce all the hallmark features of asthma in mice, and this effect of infection may account for some/all of the increases in severity of allergic airway disease. The effect of infection on lung structure was also assessed. Neonatal infection had striking and permanent effects on lung structure. Infection resulted in alveolar wall destruction, which substantially increased alveolar diameter in adulthood, which was reminiscent of emphysema in the parenchyma. These destructive effects were permanent as they were present in adult mice 9 weeks after neonatal infection. Infection had little observable effect on airway structure (thickness of airways-associated basement membrane, epithelial cell or smooth muscle layers), apart from mucus-secreting cell hyperplasia. These effects on parenchymal structure have recently been confirmed by others $^{59}$ and may contribute to the induction of AHR and reduced lung function by neonatal infection. It is not known how these specific changes in lung structure enhance AHR, which was detected by increases in transpulmonary resistance and decreases in dynamic compliance (i.e. the inverse of elastic recoil). It is likely that reductions in alveolar attachments to the airway wall decreased airway support and elastic recoil, leading to alterations in resistance and compliance. Furthermore, the level of resistance is mostly driven by the larger airways, although the smaller airways contribute, whereas the smaller airways are primarily responsible for compliance. The consequences of infection that resulted in parenchymal destruction may have led to a stiffening of the smaller airways, which could enhance their contribution to resistance and reduce compliance. It is also possible that infection of the larger airways and smooth muscle may have altered their reactivity without changing their structure, although this possibility remains untested.

We also examined the effect of chlamydial infection during the infant and adult stages. ${ }^{11}$ Infant mice were infected at 3 weeks of age just after weaning, which equates to a 2-year-old human infant, whereas adult mice were infected at 6 weeks of age. Allergic airway disease was induced 6 weeks after infection (i.e. when mice were 9 or 12 weeks old). Infant infection also increased the severity of allergic airway disease by increasing mucus-secreting cell numbers, IL-13 expression and reducing lung function. However, these effects occurred through very different mechanisms compared with neonatal infection. Infant infection increased Th2-mediated allergic inflammation (increased IL-5 and IL-13 release from MLN T cells stimulated with ovalbumin, and eosinophil influx into the airways) and systemic DC-induced IL-13 release, but had no effects on lung structure. By contrast, adult infection had no effect on lung structure and/or any of the features of allergic airway disease that were altered by neonatal and/or infant infections.
Thus, early-life infection increases the severity of allergic airway disease and potentially asthma, but occurs through different mechanisms in different age groups.

\section{Chlamydial RTI, IL-13 and asthma}

The role of IL-13 in chlamydial infections was further investigated. ${ }^{19,51}$ Chlamydia is known to grow in innate immune cells such as neutrophils and macrophages, ${ }^{60}$ and these observations were extended to show that bone marrow DCs could also be infected. ${ }^{19}$ Infection induced the production of IL-13 by DCs and increased the proliferation of ovalbumin TCR Tg T cells and promoted a Th2 phenotype. Adoptive transfer of infected and ovalbumin-pulsed DCs along with ovalbumin TCR Tg T cells into naïve adult mice increased IL-13 release into bronchoalveolar lavage fluid and AHR compared with similarly treated but uninfected DCs. ${ }^{19}$ These studies suggest that chlamydial RTI may subvert DC function to promote Th2 responses and AHR in asthma. These Th2 responses may be ineffective in removing the bacteria, which requires interferon (IFN)- $\gamma$ and Th1 responses for clearance. ${ }^{61,62}$

We have also used adult IL-13-deficient $(-/-)$ mice to demonstrate that infection and disease are markedly reduced in the absence of IL-13. ${ }^{51}$ Compared with their wild-type counterparts, IL-13-/- mice did not lose weight and had reduced infection and airway inflammation. Depletion of CD4 $+\mathrm{T}$ cells did not affect the clearance of Chlamydia in IL-13-/- mice, suggesting a lack of CD4+ T-cell involvement. We then showed that a lack of IL-13 increased the uptake of Chlamydia by macrophages and decreased the infection of lung epithelial cells (i.e. LA4 murine lower respiratory epithelial cells). ${ }^{51}$

Thus, IL-13 promotes chlamydial RTI, and as IL-13 is increased in the airways of asthmatics this may increase their susceptibility to infection and worsen the severity of asthma. We have also shown that an ongoing, rather than cleared, chlamydial RTI in adults increases the severity of asthma by inducing a phenotype resembling neutrophilic asthma that is dependent on infection-induced neutrophils. ${ }^{63}$ We have made similar observations with Haemophilus influenzae that may be driven by infection-induced IL-17/Th17 responses. ${ }^{64,65}$

\section{Other infections and the developmental origins of asthma}

Infections with respiratory syncytial virus and rhinovirus in early life have also been widely associated with the induction and exacerbation of asthma, which we have extensively reviewed recently, ${ }^{4}$ and it is not necessary to repeat the details here. This probably occurs through increases in airway inflammation and inappropriate $\mathrm{Th} 2$ responses that are induced by these infections, and which are reinforced upon re-infection. A recent study has shown that infection of 
neonatal mice with pneumonia virus of mice, the murine equivalent of respiratory syncytial virus, also increased the severity of subsequent allergic airway disease. ${ }^{66}$ This also occurred through the augmentation of Th2 responses. Again, infection was associated with the development of mucus hypersecretion and AHR, and the effects were dependent on signalling through the IL-4 receptor $\alpha .{ }^{66}$

Many studies have investigated the use of including probiotics in early life to inhibit the development of asthma and other allergic diseases. Probiotics can activate common mucosal surfaces, and effects in the gastrointestinal tract can alter responses in the airways. ${ }^{67}$ They have immune-modulating capacity and can alter DC responses, induce the development of Tregs and attenuate allergic responses. Administration of probiotics in children may have benefits in eczema but trials investigating their capacity to suppress asthma have had variable results and are, as yet, not recommended for management of allergic diseases in children. ${ }^{67,68}$

We have performed a series of studies that suggest that the Gram-positive bacteria $S$. pneumoniae may also have beneficial effects in asthma. ${ }^{12,26-29}$ Although this bacterium is a common respiratory pathogen, it is surprising that it has not been widely associated with asthma. The studies that have been performed have shown that infection suppresses peripheral eosinophilia ${ }^{69}$ and that $S$. pneumoniae vaccination reduces asthma exacerbations. ${ }^{70,71}$ Furthermore, asthmatic children do not necessarily seroconvert upon infection and have low $S$. pneumoniae antibody levels. ${ }^{72,73}$ Thus, infection or immunization with $S$. pneumoniae may boost immune responses and moderate allergic responses, thereby reducing the severity of asthma. ${ }^{12}$ We have shown that $S$. pneumoniae infection, the killed bacteria or its human vaccines, suppress the asthma phenotype in our models of allergic airway diseases. This occurs through the induction of increased numbers of regulatory $\mathrm{T}$ cells that have enhanced suppressive capacity and reduce the proliferation of Th2 cells and their release of cytokines. It is known that $S$. pneumoniae induces the production of regulatory $\mathrm{T}$ cells to a greater extent than other Gram-positive bacteria. ${ }^{74}$ Others have shown similar suppressive effects with daily gavage of mice with Lactobacillus, ${ }^{15}$ Helicobacter infection ${ }^{16}$ and a range of other bacteria. ${ }^{12}$

\section{Summary}

Infectious challenge in early life can promote the developmental origins of both health and disease, and our hypothesis is that the outcome depends on the nature and age of infection and perhaps also the timing of infection relative to allergen exposure. Respiratory viruses and some bacteria, particularly Chlamydia and Mycoplasma, are associated with the induction or increased severity of asthma in early life. In contrast, exposure to probiotics, S. pneumoniae or other bacteria may be protective. Whether the effects are beneficial or deleterious are likely to result from the phenotype of immune responses to infection, the nature of the interaction of the infectious agent with the immune response and the impact of infection on lung structure and function. Elucidating the relationships between infections, immunity and lung structure in early life is likely to identify new potential avenues for the development of therapeutic approaches in order to prevent the emergence of disease in early life. ${ }^{75}$

\section{Acknowledgements}

Our work was supported by grants from the National Health and Medical Research Council of Australia, The Australian Research Council, the Asthma Foundation of NSW and the Hunter Medical Research Institute.

\section{References}

1. Bush A. Asthma research: the real action is in children. Paediatr Respir Rev. 2005; 6, 101-110.

2. Prescott $S$. The development of respiratory inflammation in children. Paediatr Respir Rev. 2006; 7, 89-96.

3. Hansbro PM, Beagley KW, Horvat JC, Gibson PG. Role of atypical bacterial infection of the lung in predisposition/ protection of asthma. Pharmacol Ther. 2004; 101, 193-210.

4. Hansbro NG, Horvat JC, Wark PA, Hansbro PM. Understanding the mechanisms of viral induced asthma: new therapeutic directions. Pharmacol Ther. 2008; 117, 313-353.

5. Maslowski K, Mackay C. Diet, gut microbiota and immune responses. Nat Immunol. 2011; 12, 5-9.

6. Nagel G, Weinmayr G, Kleiner A, et al. Effect of diet on asthma and allergic sensitisation in the International Study on Allergies and Asthma in Childhood (ISAAC) Phase Two. Thorax. 2010; 65, 516-522.

7. Ly N, Litonjua A, Gold D, Celedón J. Gut microbiota, probiotics, and vitamin D: interrelated exposures influencing allergy, asthma, and obesity? J Allergy Clin Immunol. 2011; 127 , 1087-1094.

8. Jensen M, Collins C, Gibson P, Wood L. The obesity phenotype in children with asthma. Paediatr Respir Rev. 2011; 12, 152-159.

9. Culley FJ, Pollott J, Openshaw PJM. Age at first viral infection determines the pattern of $\mathrm{T}$ cell-mediated disease during reinfection in adulthood. J Exp Med. 2002; 196, 1381-1386.

10. Horvat J, Beagley K, Wade M, et al. Neonatal chlamydial infection induces mixed $\mathrm{T}$-cell responses that drive allergic airway disease. Am J Respir Crit Care Med. 2007; $176,556-564$.

11. Horvat J, Starkey M, Kim R, et al. Early-life chlamydial lung infection enhances allergic airways disease through agedependent differences in immunopathology. J Allergy Clin Immunol. 2010; 125, 617-625.

12. Thorburn A, Hansbro P. Harnessing regulatory $\mathrm{T}$ cells to suppress asthma: from potential to therapy. Am J Respir Cell Mol Biol. 2010; 43, 511-519.

13. Webley WC, Tilahun Y, Lay K, et al. Occurrence of Chlamydia trachomatis and Chlamydia pneumoniae in paediatric respiratory infections. Eur Respir J. 2009; 33, 360-367.

14. Barreto $M$, Bonafoni S, Barberi S, et al. Does a parent-reported history of pneumonia increase the likelihood of respiratory 
symptoms needing therapy in asthmatic children and adolescents? J Asthma. 2011; 48, 714-720.

15. Karimi K, Inman M, Bienenstock J, Forsythe P. Lactobacillus reuteri-induced regulatory $\mathrm{T}$ cells protect against an allergic airway response in mice. Am J Respir Crit Care Med. 2009; 179, 186-193.

16. Arnold I, Dehzad N, Reuter S, et al. Helicobacter pylori infection prevents allergic asthma in mouse models through the induction of regulatory T cells. J Clin Invest. 2011; 121, 3088-3093.

17. Dharmage S, Erbas B, Jarvis D, et al. Do childhood respiratory infections continue to influence adult respiratory morbidity? Eur Respir J. 2009; 33, 237-244.

18. Hansbro P, Kaiko G, Foster P. Cytokine/anti-cytokine therapy - novel treatments for asthma? Br J Pharmacol. 2011; 163, 81-95.

19. Kaiko G, Phipps S, Hickey D, et al. Chlamydia muridarum infection subverts dendritic cell function to promote Th2 immunity and airways hyperreactivity. J Immunol. 2008; 180, 2225-2232.

20. Kaiko G, Horvat J, Beagley K, Hansbro P. Immunological decision-making: how does the immune system decide to mount a helper T-cell response? Immunology. 2008; 123, 326-338.

21. Yang X, Gao X. Role of dendritic cells: a step forward for the hygiene hypothesis. Cell Mol Immunol. 2011; 8, 12-18.

22. Wynne O, Horvat J, Kim R, et al. Neonatal respiratory infection and adult re-infection: effect on GR and MR in the hippocampus in BALB/c mice. Brain Behav Immun. 2011; 25, 1214-1222.

23. Wynne O, Horvat J, Osei-Kumar A, et al. Early life infection permanently alters hippocampal gene expression in a sex specific manner. Stress. 2011; 14, 247-261.

24. Wynne O, Horvat J, Osei-Kumar A, et al. Effect of neonatal Chlamydia muridarum infection on adult hippocampal glucocorticoid and mineralcorticoid receptors. Dev Psychobiol. 2012; doi:10.1002/dev.20615.

25. Singh M, Ranjan Das R. Probiotics for allergic respiratory diseases - putting it into perspective. Pediatr Allergy Immunol. 2009; 2, 3368-3376.

26. Preston J, Thorburn A, Starkey M, et al. Respiratory Streptococcus pneumoniae infection suppresses allergic airways disease through the induction of regulatory T cells. Eur Respir J. 2011; 37, 53-64.

27. Preston J, Essilfie A, Horvat J, et al. Inhibition of allergic airways disease by immunomodulatory therapy with whole killed Streptococcus pneumoniae. Vaccine. 2007; 25, 8154-8162.

28. Thorburn A, O'Sullivan B, Thomas R, et al. Pneumococcal conjugate vaccine-induced $T$ regulatory cells suppress the development of allergic airways disease. Thorax. 2010; 65, 1053-1060.

29. Thorburn A, Foster P, Gibson P, Hansbro P. Components of Streptococcus pneumoniae suppress allergic airways disease and natural killer $\mathrm{T}$ cells by inducing regulatory $\mathrm{T}$ cells. J Immunol. 2012; in press.

30. Hilty M, Burke C, Pedro H, et al. Disordered microbial communities in asthmatic airways. PLoS One. 2010; 5, e8578.

31. Herbst T, Sichelstiel A, Schär C, et al. Dysregulation of allergic airway inflammation in the absence of microbial colonization. Am J Respir Crit Care Med. 2011; 184, 198-205.
32. Huang Y, Nelson C, Brodie E, et al. Airway microbiota and bronchial hyperresponsiveness in patients with suboptimally controlled asthma. J Allergy Clin Immunol. 2011; 127, 372-381.

33. Gnarpe J, Gnarpe H, Sundelöf B. Endemic prevalence of Chlamydia pneumoniae in subjectively healthy persons. Scand J Infect Dis. 1991; 23, 387-388.

34. Schmidt S, Muller C, Mahner B, Wiersbitzky S. Prevalence, rate of persistence and respiratory tract symptoms of Chlamydia pneumoniae infection in 1211 kindergarten and school age children. Pediatr Infect Dis J. 2002; 21, 758-762.

35. Kuo C, Jackson L, Campbell L, Grayston J. Chlamydia pneumoniae (TWAR). Clin Microbiol Rev. 1995; 8, 451-461.

36. Wark P, Johnston S, Simpson J, Hensley M, Gibson P. Chlamydia pneumoniae immunoglobulin A reactivation and airway inflammation in acute asthma. Eur Respir J. 2002; 20, 834-840.

37. Sutherland E, Martin R. Asthma and atypical bacterial infection. Chest. 2007; 132, 1962-1966.

38. Webley W, Salva P, Andrzejewski C, et al. The bronchial lavage of pediatric patients with asthma contains infectious Chlamydia. Am J Respir Crit Care Med. 2005; 171, 1083-1088.

39. Normann E, Gnarpe J, Wettergren B, et al. Association between Chlamydia pneumoniae antibodies and wheezing in young children and the influence of sex. Thorax. 2006; 61, 1054-1058.

40. von Hertzen LC. Role of persistent infection in the control and severity of asthma: focus on Chlamydia pneumoniae. Eur Respir J. 2002; 19, 546-556.

41. Cunningham A, Johnston S, Julious S, Lampe F, Ward M. Chronic Chlamydia pneumoniae infection and asthma exacerbations in children. Eur Respir J. 1998; 11, 345-349.

42. Emre U, Roblin P, Gelling M, et al. The association of Chlamydia pneumoniae infection and reactive airway disease in children. Arch Pediatr Adolesc Med. 1994; 148, 727-732.

43. Esposito S, Blasi F, Arosio C, et al. Importance of acute Mycoplasma pneumoniae and Chlamydia pneumoniae infections in children with wheezing. Eur Respir J. 2000; 16, 1142-1146.

44. Freymuth F, Vabret A, Brouard J, et al. Detection of viral, Chlamydia pneumoniae and Mycoplasma pneumoniae infections in exacerbations of asthma in children. J Clin Virol. 1999; 13, 131-139.

45. Zaitsu M. The development of asthma in wheezing infants with Chlamydia pneumoniae infection. J Asthma. 2007; 44, 565-568.

46. Wang F, He X, Baines K, et al. Different inflammatory phenotypes in adults and children with acute asthma. Eur Respir J. 2011; 38, 567-574.

47. Webley W, Hahn D. Respiratory Chlamydophyla pneumoniae resides primarily in the lower airway. Eur Respir J. 2011; 38, 994-995.

48. Holt P, Sly P. Prevention of allergic respiratory disease in infants: current aspects and future perspectives. Curr Opin Allergy Clin Immunol. 2007; 7, 547-555.

49. Brunetti L, Colazzo D, Francavilla R, et al. The role of pulmonary infection in pediatric asthma. Allergy Asthma Proc. 2007; 28, 190-193.

50. Krüll M, Bockstaller P, Wuppermann F, et al. Mechanisms of Chlamydophila pneumoniae-mediated GM-CSF release in human bronchial epithelial cells. Am J Respir Cell Mol Biol. 2006; 34, 375-382. 
51. Asquith K, Horvat J, Kaiko G, et al. Interleukin-13 promotes susceptibility to chlamydial infection of the respiratory and genital tracts. PLoS Pathog. 2011; 7, e1001339.

52. Jahn H, Krüll M, Wuppermann F, et al. Infection and activation of airway epithelial cells by Chlamydia pneumoniae. J Infect Dis. 2000; 182, 1678-1687.

53. Prochnau D, Rödel J, Hartmann M, Straube E, Figulla H. Growth factor production in human endothelial cells after Chlamydia pneumoniae infection. Int J Med Microbiol. 2004; 294, 53-57.

54. Molestina RE, Miller RD, Ramirez JA, Summersgill JT. Infection of human endothelial cells with Chlamydia pneumoniae stimulates transendothelial migration of neutrophils and monocytes. Infect Immun. 1999; 67, 1323-1330.

55. Coombes B, Mahony J. Chlamydia pneumoniae infection of human endothelial cells induces proliferation of smooth muscle cells via an endothelial cell-derived soluble factor(s). Infect Immun. 1999; 67, 2909-2915.

56. Rodel J, Woytas M, Groh A, et al. Production of basic fibroblast growth factor and interleukin 6 by human smooth muscle cells following infection with Chlamydia pneumoniae. Infect Immun. 2000; 68, 3635-3641.

57. Doganci A, Sauer K, Karwot R, Finotto S. Pathological role of IL- 6 in the experimental allergic bronchial asthma in mice. Clin Rev Allergy Immunol. 2005; 28, 257-270.

58. Wills-Karp M, Santeliz J, Karp CL. The gremless theory of allergic disease: revisiting the hygiene hypothesis. Nat Rev Immunol. 2001; 1, 69-75.

59. Jupelli M, Murthy A, Chaganty B, et al. Neonatal chlamydial pneumonia induces altered respiratory structure and function lasting into adult life. Lab Invest. 2011; 91, 1530-1539.

60. Beagley K, Huston W, Hansbro P, Timms P. Chlamydial infection of immune cells: altered function and implications for disease. Crit Rev Immunol. 2009; 29, 275-305.

61. Jupelli M, Guentzel M, Meier P, et al. Endogenous IFN\{gamma\} production is induced and required for protective immunity against pulmonary chlamydial infection in neonatal mice. J Immunol. 2008; 180, 4148-4155.

62. Jupelli M, Selby D, Guentzel M, et al. The contribution of interleukin-12/interferon-gamma axis in protection against neonatal pulmonary Chlamydia muridarum challenge. J Interferon Cytokine Res. 2010; 30, 407-415.
63. Horvat J, Starkey M, Kim R, et al. Chlamydial respiratory infection during allergen sensitization drives neutrophilic allergic airways disease. J Immunol. 2010; 184, 4159-4169.

64. Essilfie A, Simpson J, Horvat J, et al. Haemophilus influenzae infection drives IL-17-mediated neutrophilic allergic airways disease. PLoS Pathog. 2011; 7, e1002244.

65. Essilfie A, Simpson J, Dunkley M, et al. Combined Haemophilus influenzae respiratory infection and allergic airways disease drives chronic infection and features of steroid-resistant neutrophilic asthma. Thorax. 2012; in press.

66. Siegle J, Hansbro N, Herbert C, et al. Early-life viral infection and allergen exposure interact to induce an asthmatic phenotype in mice. Respir Res. 2010; 11, 14-29.

67. Forsythe P. Probiotics and lung diseases. Chest. 2011; 139, 901-908.

68. Yao T, Chang C, Hsu Y, Huang J. Probiotics for allergic diseases: realities and myths. Pediatr Allergy Immunol. 2010; 21, 900-919.

69. Bass D. Behaviour of eosinophil leukocytes in acute inflammation. Lack of dependence on adrenal function. J Clin Invest. 1975; 55, 1229-1236.

70. Schuller D. Prophylaxis of otitis media in asthmatic children. Pediatr Infect Dis. 1983; 2, 280-283.

71. Ansaldi F, Turello V, Lai P, et al. Effectiveness of a 23-valent polysaccharide vaccine in preventing pneumonia and noninvasive pneumococcal infection in elderly people: a large-scale retrospective cohort study. J Int Med Res. 2005; 33, 490-500.

72. Rose M, Schubert R, Kujumdshiev S, Kitz R, Zielen S. Immunoglobulins and immunogenicity of pneumococcal vaccination in preschool asthma. Int J Clin Pract. 2006; 60, 1425-1431.

73. Lee H, Kang J, Henrichsen J, et al. Immunogenicity and safety of a 23-valent pneumococcal polysaccharide vaccine in healthy children and in children at increased risk of pneumococcal infection. Vaccine. 1995; 13, 1533-1538.

74. Kadioglu A, Coward W, Colston M, Hewitt C, Andrew P. CD4-T-lymphocyte interactions with pneumolysin and pneumococci suggest a crucial protective role in the host response to pneumococcal infection. Infect Immun. 2004; 72, 2689-2697.

75. Szefler S. Advances in pediatric asthma in 2010: addressing the major issues. J Allergy Clin Immunol. 2011; 127, 102-115. 